\title{
26423 - HEART RATE VARIABILITY TO PREDICT POSTOPERATIVE NAUSEA AND VOMITING
}

\author{
William Li Pi Shan MD, Roupen Hatzakorzian, MD; Thomas Schricker, MD PhD; \\ Steven Backman, Alain Deschamps, MD PhD; McGill University Health Centre, \\ Montreal, QC, Canada
}

\section{INTRODUCTION:}

Despite recent advances in pharmacological treatment (3), the incidence of postoperative nausea and vomiting (PONV) is significant $(20-30 \%)(1,2)$. PONV is associated with a few patient characteristics (3). There is currently however no method to predict PONV in an individual patient about to undergo surgery. The autonomic nervous system is implicated in the physiology of nausea, and an increase in baseline parasympathetic activity may be associated with a high risk of PONV $(4,5)$. Heart rate variability (HRV) and blood pressure variability (BPV) are non-invasive tools to measure autonomic drive. We hypothesize that patients who exhibit a high parasympathetic drive have an increased risk of PONV.

METHODS:

Following REB approval and patient consent, ECG and non-invasive continuous blood pressure measurement were obtained preoperatively in 21 women undergoing abdominal hysterectomy. Wavelet transform was used for analysis of HRV and BPV. Five wavelet coefficients were calculated (21 to 25) for HRV and BPV. Lower coefficients correspond to high frequency power and higher coefficients to low frequency power. All patients received a standard anesthetic. Anti-emetic prophylaxis and neuraxial techniques were avoided. PONV was assessed for $24 \mathrm{hrs}$ following surgery.

RESULTS:

Fifteen patients (71.4\%) experienced PONV. Cumulative wavelet coefficients for $20 \mathrm{~min}$ were statistically analyzed using the Mann-Whitney test for non-parametric data (Table 1). Using the HF range of HRV, we found that the mean cumulative wavelet coefficient in the nausea group was significantly greater $(p<0.0001)$ than the no nausea group. The $\mathrm{LF}$ range also showed a significant difference $(\mathrm{p}<0.0001)$ between the nausea group and the no nausea group. The HF range of HRV corresponds to predominant parasympathetic activity. The BPV analysis showed that in the high frequency range the mean blood pressure cumulative wavelet coefficient differed between both groups $(\mathrm{p}<0.0007)$ with the wavelet coefficients in the no nausea group being significantly greater than the nausea group (Table 1). The HF range of BPV corresponds to predominant sympathetic activity. DISCUSSION:

Our preliminary results suggest an increased parasympathetic tone in patients with nausea and an increased sympathetic tone in patients without nausea. Using a non-invasive baseline measurement of HRV and BPV, it may be possible to predict those patients at significant risk of developing PONV. Further studies with a cutoff value to predict which patients may be at high risk of developing PONV are needed to validate this method. REFERENCES:

1. Anesth Analg 1994; 78:7-16

2. Anesthesiology Clin N Am 2002; 20: 709-722

3. NEJM 350; 24: 2441-2451 
4. Psychophysiology 2003; 40(1): 39-44

5. Anesth Analg 2002; 95: 133-43

\begin{tabular}{|c|c|c|c|}
\hline & \multicolumn{2}{|c|}{ Mean Wavelet coefficient of Heart rate variability (SD) } & \multirow[b]{2}{*}{$P$ value } \\
\hline & Nausea & No nausea & \\
\hline $\mathrm{HF}$ & $113526.1(119859) \mathrm{sec}^{2}$ & $43671.8(52138)$ & $\mathrm{P}<0.0001$ \\
\hline \multirow[t]{3}{*}{ LF } & $72887.5(73847) \quad \mathrm{sec}^{2}$ & $26418.8(26001) \quad \sec ^{2}$ & $\mathrm{P}<0.0001$ \\
\hline & \multicolumn{2}{|c|}{$\begin{array}{l}\text { Mean Wavelet Coefficient of Blood pressure variability } \\
\text { (SD) }\end{array}$} & \\
\hline & Natusea & No natsea & P value \\
\hline HF mean BP & $421.0(372.3)$ & $647.1(595.4)$ & $\mathrm{P}<0.0007$ \\
\hline
\end{tabular}

Table1: Differences between the natsea group $(\mathrm{N}=15)$ and no nansea grotm $(\mathrm{N}=6)$,

sbowing the differences in mesn wavelet coefficients and their respective p-values (using Mann-Whitney test) 